\title{
Minimum Alveolar Concentration-Awake of
}

\section{Sevoflurane is Decreased in Patients with}

\section{Parkinson's Disease: An Up-and-Down Sequential Allocation Trial}

This article was published in the following Dove Press journal:

Clinical Interventions in Aging

\author{
Chengwei Yang ${ }^{1, *}$ \\ Fang Kang ${ }^{1, *}$ \\ Wenjun Meng' \\ Meirong Dong' \\ Xiang Huang' \\ Sheng Wang' \\ Zhiyi Zuo ${ }^{2}$ \\ Juan Li (iD)
}

'Department of Anesthesiology, The First Affiliated Hospital of USTC, Division of Life Sciences and Medicine, University of Science and Technology of China, Hefei, Anhui, People's Republic of China; ${ }^{2}$ Department of Anesthesiology,

University of Virginia, Charlottesville, Virginia, USA

*These authors contributed equally to this work
Correspondence: Zhiyi Zuo

Department of Anesthesiology, University of Virginia, Charlottesville, Virginia 22908, USA

Email zz3c@virginia.edu

Juan $\mathrm{Li}$

Department of Anesthesiology, The First Affiliated Hospital of USTC, Division of Life Sciences and Medicine, University of Science and Technology of China, Hefei 230036, People's Republic of China Email huamuzil999@I63.com
Background: An increasing number of patients with Parkinson's disease (PD) will have surgery under general anesthesia. A previous study demonstrated that propofol requirement for inducing unconsciousness in PD patients was lower than that in non-PD (NPD) patients. However, the requirement of inhaled anesthetics in PD patients has not been clarified. The aim of this study was to investigate the minimum alveolar concentration-awake $\left(\mathrm{MAC}_{\text {awake }}\right)$ of sevoflurane in patients with PD compared to NPD patients.

Patients and Methods: The current study is an up-and-down sequential allocation trial. The initial end-tidal concentration of sevoflurane (CETsevo) was estimated by the response of the previous patient to verbal command using the Dixon's up-and-down method. The first patient in each group received CETsevo at $1 \%$, and the step size between patients was $0.2 \%$.

Results: Forty-one patients including 20 PD patients and 21 NPD patients were enrolled. Patients' characteristics and arterial blood gas parameters (except blood sodium) were comparable between two groups. The $\mathrm{MAC}_{\text {awake }}$ of sevoflurane estimated by the Dixon's upand-down method in PD patients $(0.47 \% \pm 0.08 \%$ [Mean \pm S.D.]) was significantly lower than that in NDP patients $(0.64 \% \pm 0.10 \%)(P=0.003)$. The estimated difference in means was $0.17 \%$ (95\% CI, $0.10-0.24 \%$ ). Probit analysis showed that the $\mathrm{MAC}_{\text {awake }}$ of sevoflurane in PD and NPD patients was $0.49 \%$ (95\% CI, 0.42-0.57\%) and $0.67 \%$ (95\% CI, 0.59-$0.76 \%$ ), respectively. The relative median potency was 0.73 (95\% CI, 0.38-0.94).

Conclusion: Patients with PD exhibit a significantly lower $\mathrm{MAC}_{\mathrm{awake}}$ of sevoflurane compared with NPD patients. Clinicians should avoid an overdose of sevoflurane in patients with PD.

Trial Registration: Registered at ChiCTR1900026956.

Keywords: Parkinson's disease, sevoflurane, the minimum alveolar concentration-awake

\section{Introduction}

Parkinson's disease (PD) is the second common neurodegenerative disorder at present. ${ }^{1}$ It was reported that the prevalence of PD is about $0.3 \%$ in the general population and $1 \%$ in the people older than 60 years. ${ }^{2}$ Moreover, the incidence of PD is growing quickly over the last 20 years. ${ }^{3}$ Symptoms of PD mainly include bradykinesia, rigidity, and resting tremor, which affect the quality of life of patients significantly. 
An increasing number of patients with PD will have surgery under general anesthesia as the society is aging rapidly. We have noticed that patients with $\mathrm{PD}$ were slower to emerge from anesthesia compared with non-PD (NPD) patients in clinical practice. However, very little is known about the requirements of PD patients for anesthetic agents. $\mathrm{Xu}$ et al found that propofol requirement to induce unconsciousness in PD patients was lower than NPD patients due to the degenerative brain. ${ }^{4}$ The reasons for this decreased requirement might include the reduced dopamine production and anti-PD medications in PD patients. Their team also demonstrated that patients with PD have lower remifentanil requirements to suppress the response to tracheal intubation and skin incision. ${ }^{5}$ Sevoflurane is one of the most commonly used anesthetic agents in clinic anesthesia. However, the requirement of sevoflurane in PD patients is unknown. Proper dose of sevoflurane avoids deep general anesthesia, which may affect the practice of the enhanced recovery after surgery (ERAS) of PD patients. ${ }^{6,7}$

The minimum alveolar concentration (MAC) to achieve a $50 \%$ probability of no response to a verbal command ( $\left.\mathrm{MAC}_{\mathrm{awake}}\right)$ can be used to measure the hypnotic potency of inhaled anesthetics. ${ }^{8}$ We hypothesized that the $\mathrm{MAC}_{\text {awake }}$ of sevoflurane was decreased in patients with PD compared with those with normal neurological function.

\section{Patients and Methods}

The current study is an up-and-down sequential allocation trial. Ethical approval for this study (2019KY111) was provided by the Medical Research Ethic Committee of Anhui Provincial Hospital, Hefei, China (Chairperson Prof Zuojun Shen) on 14 October 2019. Written informed consent was obtained from all subjects participating in the trial. The trial was registered prior to patient enrollment at ChiCTR1900026956, Principal investigator: Chengwei Yang, Date of registration: October 14, 2019. We performed this study from October 15, 2019 to May 26, 2020. Written informed consents were obtained from all participants. Thirty patients with PD undergoing bilateral deep brain stimulation (DBS) electrode insertion and pulse generator placement were included in this study. Thirty patients with normal neurological function scheduled for supratentorial brain tumor (less than $30 \mathrm{~mm}$ in diameter) resection (NPD) served as controls. All patients were 40 to 64 years old. Exclusionary criteria were as follows: an American Society of Anesthesiologists (ASA) score higher than class III, a body mass index (BMI) more than $30 \mathrm{~kg}$. $\mathrm{m}^{-2}$, a history of respiratory, hepatic, renal, or psychiatric diseases, cardiac ejection fraction less than $40 \%$, a history of alcohol or drug abuse, contraindication to inhalational drugs, history of emergence delirium with sevoflurane induction, medications known to affect $\mathrm{MAC}_{\text {awake, }}$, known family history of malignant hyperthermia, a history of auditory impairment, acid-base or electrolyte abnormalities, diabetes, sepsis, or obvious weight loss caused by carcinoma.

After DBS electrodes were inserted under local anesthesia, we conducted general anesthesia to place the pulse generator in PD patients. All patients were fasted for 8 hours and took no premedication. PD patients stopped taking anti-PD drugs at the night before surgery. After arriving in the operation room, a $20 \mathrm{G}$ vena catheterization was established. Patients received $10 \mathrm{~kg} \cdot \mathrm{mL}^{-1}$ lactated Ringer's solution and maintained at a rate of $10 \mathrm{~mL}$. $\mathrm{kg}^{-1} \cdot \mathrm{h}^{-1}$. The temperature in the operation was controlled between $24^{\circ} \mathrm{C}$ and $26^{\circ} \mathrm{C}$. Electrocardiograms, pulse oxygen saturation and invasive arterial pressures were routinely monitored. Inspired and expired concentrations of sevoflurane and oxygen and end-tidal carbon dioxide $\left(\mathrm{ETCO}_{2}\right)$ were continuously monitored using a Drager Primus machine (Draegerwerk AG \& Co. KGaA, Lübeck, Germany) calibrated by a standard gas mixture before induction of anesthesia. An arterial blood gas analysis was conducted in each patient. PD patients received a DBS electrode insertion under local anesthesia using $30 \mathrm{~mL} 1 \%$ lidocaine and $0.375 \%$ ropivacaine mixture. Then, we performed general anesthesia to place the pulse generator. NPD patients received local anesthetics of the same volume before induction of anesthesia.

The $\mathrm{MAC}_{\text {awake }}$ of sevoflurane (HengRui, Pharmaceutical Co, Ltd., Shanghai, China) was estimated using the Dixon's up and down method according to previous studies. ${ }^{8,9}$ A mixed gas with $8 \%$ sevoflurane and $100 \%$ oxygen at a flow rate of $6 \mathrm{~L} / \mathrm{min}$ primed the circuit for 1 minute. Then, we asked patients to take deep breaths via a tight-fitting facemask attached to a semiclosed circuit system (Vapor 2000, Draegerwerk AG \& Co. KGaA, Lübeck, Germany). If the $\mathrm{ETCO}_{2}$ greater than $45 \mathrm{mmHg}$ or tidal volume less than $500 \mathrm{~mL}$, mechanical ventilation was applied. After the patient lost responsiveness, the concentration of sevoflurane was adjusted to a predetermined level. The first patient in each group received an end-tidal concentration of sevoflurane (CETsevo) at 1\%. After the CETsevo was 
maintained for 15 minutes, an anesthesiologist blinded to the study design asked the patient to open eyes in a normal tone for no more than 3 times. If the patient made a response appropriately, the CETsevo for the next patient was increased by a step of $0.2 \%$. In contrast, if the patient made no response, the CETsevo for the next patient was decreased by $0.2 \%$. The study was continued until 7 "no response/response" crossover pairs had occurred.

Another anesthesiologist blinded to the study design recorded the data. A bolus of 6-10 $\mathrm{mg}$ ephedrine was injected intravenously if the mean arterial pressure (MAP) was less than $60 \mathrm{mmHg}$ or reduced by more than $20 \%$ of baseline values. Atropine $0.5 \mathrm{mg}$ was administered intravenously if the heart rate (HR) was less than 50 beats per minute. After finishing this study, we deepened the anesthesia to perform tracheal intubation and surgical procedure.

\section{Statistical Analysis}

Statistical analysis was performed using SPSS version 20.0 software (SPSS Inc., Chicago, IL). Data are expressed as the means \pm standard deviations for continuous variables or the number (percentage) for categorical variables. The $\mathrm{MAC}_{\text {awake }}$ of sevoflurane was estimated by calculating the mean of 7 independent crossover pairs

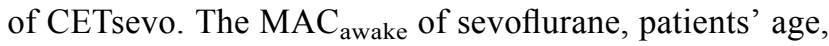
weight, and arterial blood gas values between two groups were compared by the independent-samples $t$-test. Patients' sex was compared by Pearson's chi-square test. The up and down data were also analyzed using the probit analysis, ${ }^{9,10}$ deriving the $\mathrm{MAC}_{\text {awake }}$ with $95 \%$ confidence interval $(\mathrm{CI})$. The relative median potency estimated from the probit analysis, which is the ratio of CETsevo needed to obtain a 50\% probability of being awake in each group, was used to compare the calculated $\mathrm{MAC}_{\text {awake }}$ between two groups. Finally, we assessed the association between $\mathrm{MAC}_{\text {awake }}$ and $\mathrm{PD}$ (vs non-PD) while controlling for sevoflurane by a binary logistic regression analysis.

The sample size was calculated according to a previous study. ${ }^{9}$ We hypothesized that the $\mathrm{MAC}_{\text {awake }}$ of sevoflurane in PD and NPD patients were $0.5 \%$ and $0.7 \%$, respectively. The standard deviation was $0.2 \%$. Thus, in order to achieve a power of $80 \%$ and a type I error of 0.05 to detect a difference of $0.2 \%$ with a possible dropout rate of $15 \%, 19$ patients per group were required.

\section{Results}

As shown in Figure 1, 41 patients including 20 PD patients and 21 NPD patients were enrolled in the final analysis. Patients' characteristics and arterial blood gas parameters (except blood sodium) were comparable between two groups (Table 1). The anti-Parkinson medications taken by PD patients are shown in Table 2 .

The sequences for response to verbal command in two groups are shown in Figure 2. The $\mathrm{MAC}_{\text {awake }}$ of sevoflurane estimated by the Dixon's up-and-down method in PD patients $(0.47 \% \pm 0.08 \%$ [Mean \pm S.D.] ) was significantly lower than that in NDP patients $(0.64 \% \pm 0.10 \%)$ $(P=0.003)$. The estimated difference in means was $0.17 \%$ (95\% CI, 0.10-0.24\%). From the probit analysis, the $\mathrm{MAC}_{\text {awake }}$ of sevoflurane in PD and NPD patients were $0.49 \%$ (95\% CI, 0.42-0.57\%) and $0.67 \%$ (95\% CI, 0.59 $0.76 \%$ ), respectively. The relative median potency was 0.73 (95\% CI, 0.38-0.94), the CI of which did not include one, indicating a difference in the median potency between two groups (Table 3). Figure 3 showed the dose-response curve of the probability of being awake in two groups from the probit analysis. The age, weight, and sex were not significantly different between "awake" and "not awake" patients (Table 4). Table 3 demonstrated that PD patients had a significant lower risk of being awake compared with NPD patients when controlling for age, weight, and sex $(\mathrm{OR}=0.041667,95 \%$ CI, 0.003441-0.504522, $P=0.0125$ ) (Table 5).

One PD patient' HR was less than 50 beats per minute, which was successfully treated with $0.5 \mathrm{mg}$ atropine.

\section{Discussion}

The current study demonstrated that the $\mathrm{MAC}_{\text {awake }}$ of sevoflurane in patients with Parkinson's disease was significantly lower than that in NPD patients. We derived the $\mathrm{MAC}_{\text {awake }}$ of NPD patients as $0.64 \%$, which is similar to those in previous studies. ${ }^{9,11}$

The Dixon's up-and-down method is widely used to estimate the MAC of inhaled anesthetics. ${ }^{9,12-14}$ At least 6 crossover pairs are required when using the Dixon's method. ${ }^{15}$ Based on the calculated sample size, this study was continued until 7 no response/response crossover pairs had occurred. In the current study, a high concentration of sevoflurane was firstly used to make patients lose responsiveness. Then, we adjusted the concentration of sevoflurane to a predetermined level to test the responsiveness of 


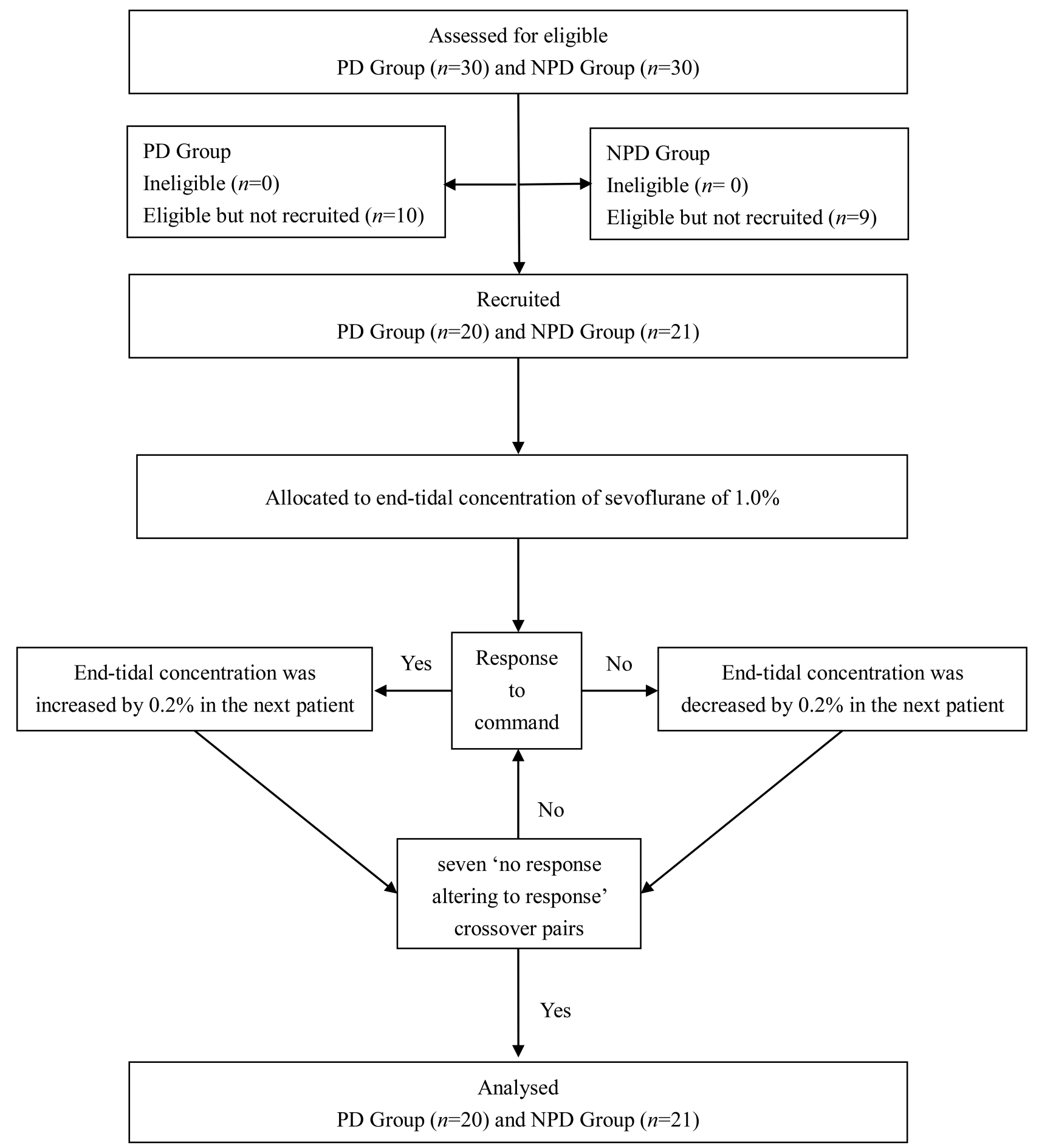

Figure I Flow diagram for the Dixon's up-and-down method.

Abbreviations: PD, Parkinson's disease; NPD, non-Parkinson's disease

patients. This method is widely used to investigate the $\mathrm{MAC}_{\text {awake }}$ of sevoflurane. ${ }^{9,14,16}$

To our best knowledge, this is the first trial to explore the MAC of inhaled anesthetic in patients with PD. In our hospital, more than $70 \%$ PD patients undergoing DBS surgery are younger than 65 yr. Thus, we chose middleaged (40-64 yr) patients in our study. The PD patients' average age was $57.2 \mathrm{yr}$, which is similar to that in a previous study (57.4 yr) by Wang et al in China. ${ }^{5}$ The time required to reach an equilibrium between alveolar and 
Table I Patients' Characteristics and Arterial Blood Gas Results

\begin{tabular}{|c|c|c|c|}
\hline Parameters & $\begin{array}{l}\text { PD Group } \\
(n=20)\end{array}$ & $\begin{array}{l}\text { NPD Group } \\
(n=2 I)\end{array}$ & $P$ \\
\hline Age, yr & $57.2 \pm 6.7$ & $54.8 \pm 6.0$ & 0.236 \\
\hline Sex, male/female & $9 / 11$ & $10 / 11$ & 0.867 \\
\hline Height, cm & $161.8 \pm 8.0$ & $163.4 \pm 5.8$ & 0.463 \\
\hline Weight, kg & $60.1 \pm 6.9$ & $62.8 \pm 6.9$ & 0.215 \\
\hline Temperature, ${ }^{\circ} \mathrm{C}$ & $36.4 \pm 0.2$ & $36.4 \pm 0.2$ & 0.600 \\
\hline $\mathrm{pH}$ & $7.4 I \pm 0.03$ & $7.41 \pm 0.02$ & 0.577 \\
\hline $\mathrm{PaO}_{2}(\mathrm{mmHg})$ & $85.2 \pm 4.2$ & $86.6 \pm 3.3$ & 0.216 \\
\hline $\mathrm{PaCO}_{2}(\mathrm{mmHg})$ & $37.7 \pm 1.5$ & $37.8 \pm 2.6$ & 0.882 \\
\hline $\mathrm{Na}^{+}(\mathrm{mmol} / \mathrm{L})$ & $139.7 \pm 2.5$ & $138.2 \pm 1.7$ & $0.033^{*}$ \\
\hline $\mathrm{K}^{+}(\mathrm{mmol} / \mathrm{L})$ & $3.9 \pm 0.4$ & $3.8 \pm 0.2$ & 0.285 \\
\hline $\mathrm{Ca}^{2+}(\mathrm{mmol} / \mathrm{L})$ & $1.10 \pm 0.03$ & $1.09 \pm 0.03$ & 0.512 \\
\hline Glucose (mmol/L) & $5.5 \pm 0.6$ & $5.8 \pm 0.5$ & 0.081 \\
\hline Lactic acid (mmol/L) & $1.55 \pm 0.26$ & $1.60 \pm 0.31$ & 0.474 \\
\hline Hemoglobin $(g / L)$ & $119.3 \pm 11.0$ & $117.7 \pm 10.7$ & 0.643 \\
\hline Hematocrit (\%) & $0.35 \pm 0.03$ & $0.34 \pm 0.03$ & 0.373 \\
\hline
\end{tabular}

Notes: Data are presented as mean \pm S.D. or number of subjects. $*$ The $P$ value for significance tested by $t$ test is set at $<0.05$.

Abbreviations: PD, Parkinson's disease; NPD, non-Parkinson's disease.

target organ concentrations is 9-12 minutes. ${ }^{9,17}$ Thus, we maintained the end-tidal sevoflurane concentration for 15 minutes before we assessed the response of subjects. Many factors influence the efficacy of inhaled anesthetics, including age, ${ }^{18,19}$ temperature, ${ }^{20}$ hypotension, acid-base status, ${ }^{21}$ diabetes, ${ }^{22}$ and sepsis. ${ }^{23}$ We excluded the patients with these pathophysiologic conditions. Moreover, patients' characteristics and blood gas values were
Table 2 The Anti-Parkinson Medications Taken by Patients with Parkinson's Disease

\begin{tabular}{|l|l|}
\hline Medications & Number of Uses (\%) \\
\hline Levodopa/benserazide & $20(100 \%)$ \\
Amantadine & II (55\%) \\
Piribedil & $5(25 \%)$ \\
Trihexyphenidyl & $3(15 \%)$ \\
Selegiline & $2(10 \%)$ \\
Levodopa/carbidopa & $\mathrm{I}(5 \%)$ \\
Pramipexole & $\mathrm{I}(5 \%)$ \\
\hline
\end{tabular}

Note: Data are presented as number (percentages).

generally comparable, making our results more reliable in measuring the effects of $\mathrm{PD}$ on $\mathrm{MAC}_{\text {awake }}$.

There may be several reasons why PD patients have a lower $\mathrm{MAC}_{\text {awake }}$ value of sevoflurane compared with NPD patients. Previous studies have demonstrated that PD patients show increased $\gamma$-aminobutyric acid (GABA) in several brain regions ${ }^{24-26}$ and decreased cortical glutamate ${ }^{27}$ by using the magnetic resonance spectroscopy. GABA and glutamate are major inhibitory and excitatory neurotransmitters in the brain. Sevoflurane at amnestic concentrations suppressed synaptic plasticity by enhancing GABA-mediated inhibition and reducing glutamate release. ${ }^{28}$ Intrathecal injection of a $\mathrm{GABA}_{\mathrm{A}}$ agonist or a glutamate antagonist induced a significant reduction of sevoflurane MAC in rats. ${ }^{29} \mathrm{Xu}$ et al found that propofol requirement to induce
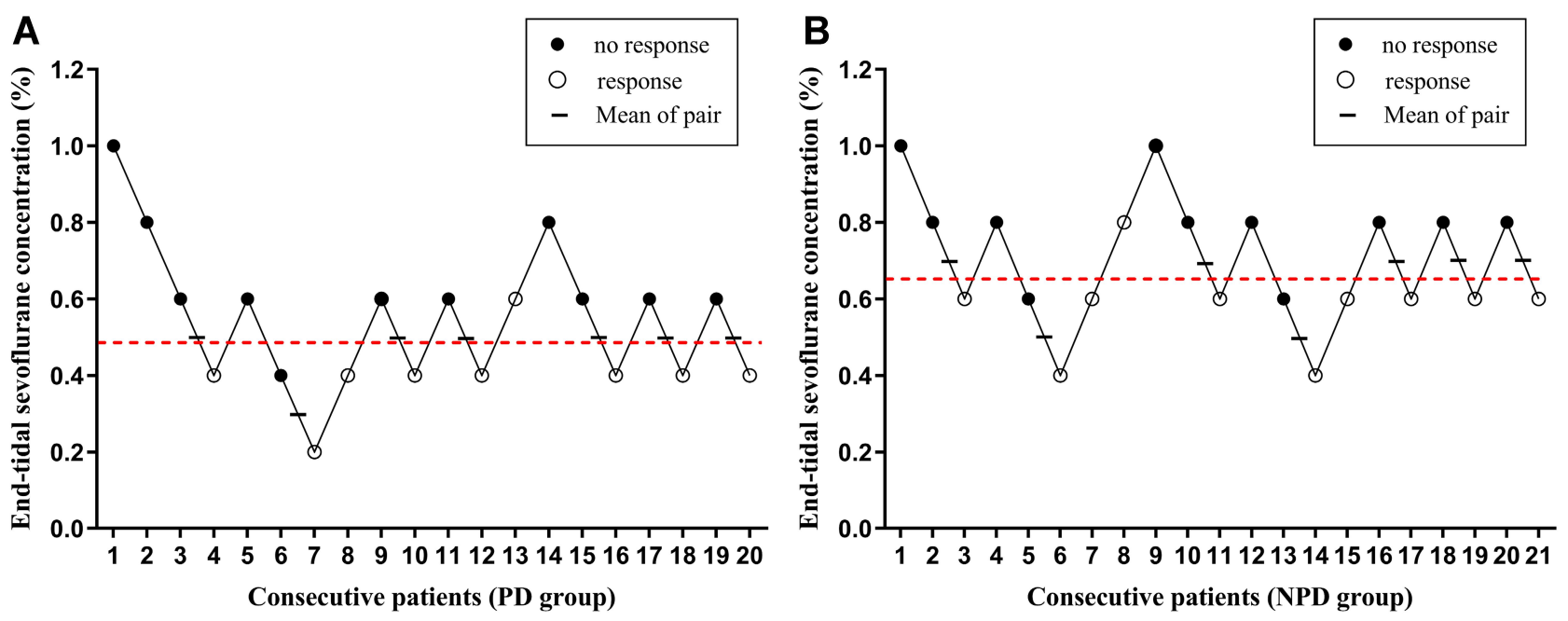

Figure 2 Assessment of responses to verbal command under a predetermined end-tidal concentration of sevoflurane using the Dixon's up-and-down method in 20 consecutive PD patients (A) and 21 consecutive NPD patients (B). A "no response" concentration is denoted by a solid circle; a "response" concentration is denoted by an open circle; horizontal bars represent crossover midpoints ("no response" to "response"). The MAC $_{\text {awake }}$ values are indicated with red dashed line. Abbreviations: PD, Parkinson's disease; NPD, non-Parkinson's disease. 
Table 3 Minimum Alveolar Concentration-Awake of Sevoflurane

\begin{tabular}{|l|l|l|l|l|l|}
\hline Methods & $\begin{array}{l}\text { PD Group (n=20) } \\
(\%)\end{array}$ & $\begin{array}{l}\text { NPD Group (n=2 I) } \\
\mathbf{( \% )}\end{array}$ & $\begin{array}{l}\text { Differences (95\% CI) } \\
\mathbf{( \% )}\end{array}$ & $\boldsymbol{p}$ & $\begin{array}{l}\text { Relative Median } \\
\text { Potency }\end{array}$ \\
\hline $\begin{array}{l}\text { Dixon's method } \\
\text { Probit analysis (95\% Cl) }\end{array}$ & $\begin{array}{l}0.47 \pm 0.08 \\
0.49(0.42-0.57)\end{array}$ & $\begin{array}{l}0.64 \pm 0.10 \\
0.67(0.59-0.76)\end{array}$ & $\begin{array}{l}0.17(0.10-0.24) \\
-\end{array}$ & $\begin{array}{l}0.003 * \\
-\end{array}$ & $0.73(0.38-0.94)$ \\
\hline
\end{tabular}

Notes: Data from the Dixon's up-and-down method are presented as mean \pm S.D. Date from the probit analysis are $E C_{50}$ with $(95 \%$ confidence interval). $*$ The $P$ value for significance tested by $t$ test is set at 0.05 .

Abbreviations: PD, Parkinson's disease; NPD, non- Parkinson's disease; $\mathrm{Cl}$, confidence interval; $\mathrm{EC}_{50}$, median effective concentration.

unconsciousness was reduced in patients with PD. ${ }^{4}$ Thus, the widely used intravenous and inhaled anesthetics were both reduced in PD patients. Propofol is known to potentiate activity of the inhibitory neurotransmitter GABA. ${ }^{30}$ Thus, the altered neurotransmitters, especially GABA, may play important roles in the reduction of anesthetics in PD patients. In addition, dopamine levels in the brain might indirectly affect the inhaled anesthetic requirements. A previous study reported that a dopamine antagonist led to a decreased halothane MAC. ${ }^{31}$ The mice with high dopamine levels in the mesencephalon exhibited an increased enflurane requirement. ${ }^{32}$ Therefore, depletion of central nervous system dopamine in $\mathrm{PD}$ patients might decrease the $\mathrm{MAC}_{\text {awake }}$ of sevoflurane. Moreover, some anti-Parkinson medications may decrease sevoflurane $\mathrm{MAC}_{\text {awake. Segal and his col- }}$ leagues found that levodopa dose-dependently decreased halothane MAC to a maximum of $49 \%$ of control. ${ }^{33}$ Preoperative oral amantadine, a non-competitive NMDA antagonist, reduced the inspired isoflurane concentration during maintenance of anesthesia in female patients undergoing abdominoplasty. ${ }^{34}$ In addition, piribedil and pramipexole may lead to somnolence, which might also contribute to the lower $\mathrm{MAC}_{\text {awake }}$ of sevoflurane in PD patients.

There are limitations in our study. First, our sample size is relatively small. Thus, we cannot investigate the influence of some confounding factors, including patients'

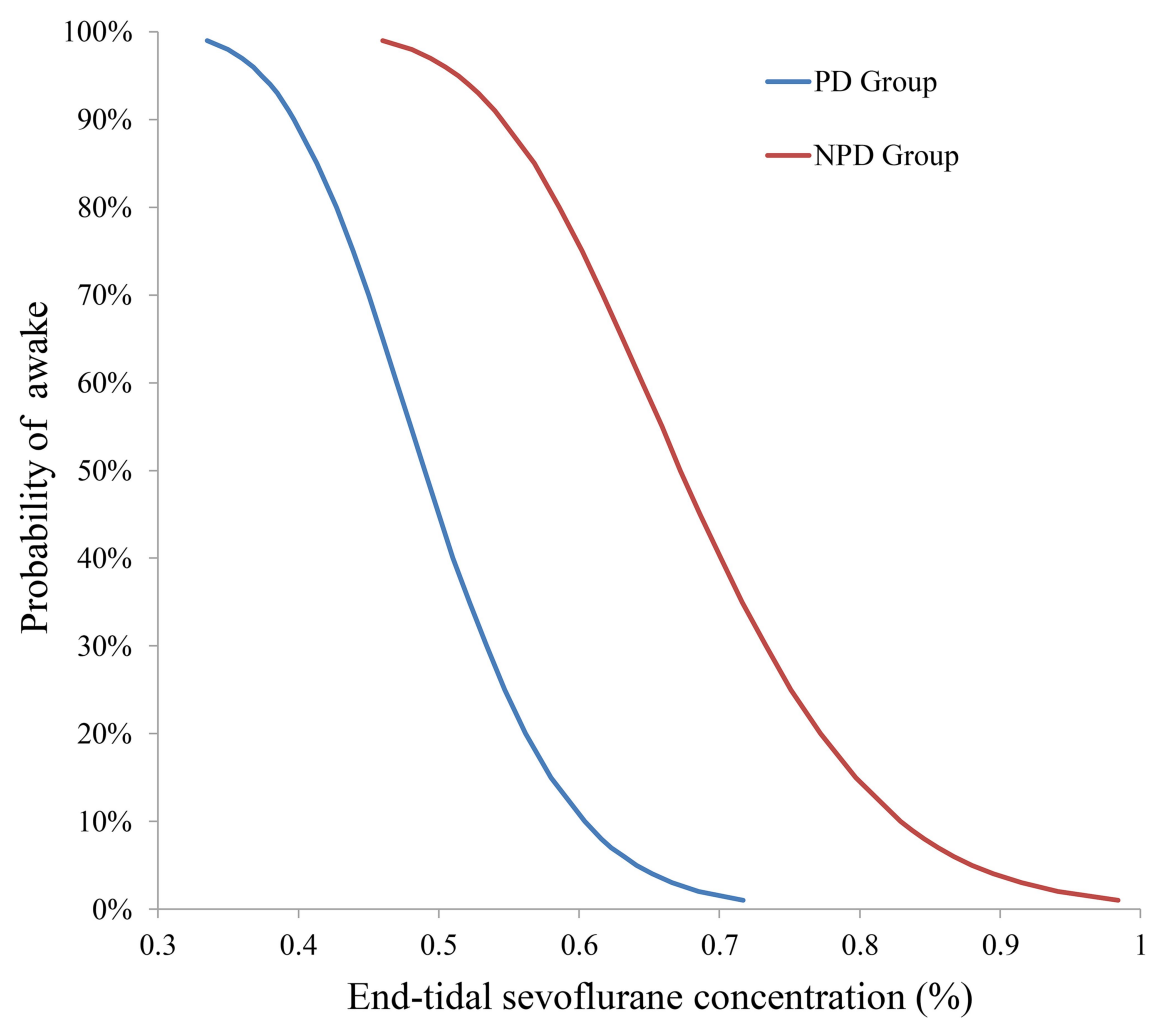

Figure 3 Dose-response curve from the probit analysis. The minimum alveolar concentration-awake ( $\mathrm{MAC}_{\text {awake }}$ ) of sevoflurane in PD patients was $0.49 \%$ ( $95 \% \mathrm{Cl}$, $0.42-0.57 \%) . M A C_{\text {awake }}$ of sevoflurane in the NPD patients was $0.67 \%(95 \% \mathrm{Cl}, 0.59-0.76 \%)$.

Abbreviations: PD, Parkinson's disease; NPD, non-Parkinson's disease. 
Table 4 The Age, Weight, and Sex Between"awake" and "Not Awake" Patients

\begin{tabular}{|l|l|l|l|}
\hline Parameters & Awake Group (n=20) & Not Awake Group (n=21) & $P$ \\
\hline Age, yr & $56.7 \pm 6.3$ & $55.2 \pm 6.5$ & 0.457 \\
Sex, male/female & $8 / 11$ & $11 / 11$ & 0.613 \\
Weight, kg & $61.6 \pm 5.9$ & $61.4 \pm 7.9$ & 0.904 \\
\hline
\end{tabular}

Note: Data are presented as mean \pm S.D. or number of subjects.

Table 5 Binary Logistic Regression Analysis for Being Awake

\begin{tabular}{|l|l|l|l|l|}
\hline Covariates (n=4I) & OR & 95\% Cl for OR & Upper \\
\cline { 3 - 5 } & & Lower & 0.000247 \\
\hline Sevoflurane concentration (\%) & $1.714 \times 10^{-8}$ & $1.187 \times 10^{-12}$ & 0.504522 & $0.0003^{2}$ \\
PD group & 0.041667 & 0.003441 & - & $0.0125^{*}$ \\
NPD group (as reference) & - & - & - \\
\hline
\end{tabular}

Notes: $*$ The $P$ value for significance tested by binary logistic regression analysis is set at $<0.05$.

Abbreviations: PD, Parkinson's disease; NPD, non-Parkinson's disease.

comorbidities and medications. Second, two groups of patients had taken different types of surgery. As DBS implantation is not performed in NPD patients, we chose patients with brain tumors as control. Thus, we cannot exclude the effect of difference in surgical procedures on the $\mathrm{MAC}_{\text {awake }}$ of sevoflurane. Third, the plasma concentrations of local anesthetics in the two study groups were not measured in our study. However, the similar $\mathrm{MAC}_{\text {awake }}$ of sevoflurane in NPD patients with previous studies indicates that the local anesthesia may have a minimal effect on the results.

\section{Conclusion}

Our study demonstrated that patients with Parkinson's disease exhibit a significant lower $\mathrm{MAC}_{\text {awake }}$ of sevoflurane compared with NPD patients. This result suggests that the sevoflurane requirement is decreased in patients with PD. Clinicians should avoid an overdose of sevoflurane in patients with PD. It may affect the practice of ERAS of these patients. More animal and clinical studies are needed to explore the underlying mechanisms of the $\mathrm{MAC}_{\text {awake }}$ reduction in these patients.

\section{Data Sharing Statement}

The data supporting this study are available from the corresponding author for a reasonable request.

\section{Ethics Approval}

The current study was conducted in accordance with the Declaration of Helsinki and approved by the Medical
Research Ethic Committee of Anhui Provincial Hospital (2019KY111).

\section{Acknowledgment}

The authors thank Yun Wu, M.D., from the Department of Anesthesiology, Second Affiliated Hospital of Anhui Medical University, for her helpful comments regarding the anesthesia induction.

\section{Funding}

This study was supported by the Natural Science Foundation of Anhui Province of China (1908085MH251) and the Fundamental Research Funds for the Central Universities (WK9110000059).

\section{Disclosure}

The authors declare that they have no conflicts of interest.

\section{References}

1. Draoui A, El Hiba O, Aimrane A, El Khiat A, Gamrani H. Parkinson's disease: from bench to bedside. Rev Neurol. 2020;176:543-559. doi:10.1016/j.neurol.2019.11.002

2. Tysnes OB, Storstein A. Epidemiology of Parkinson's disease. J Neural Transm. 2017;124(8):901-905. doi:10.1007/s00702-0171686-y

3. Collaborators GBDPsD. Global, regional, and national burden of Parkinson's disease, 1990-2016: a systematic analysis for the global burden of disease study 2016. Lancet Neurol. 2018;17(11):939-953. doi:10.1016/S1474-4422(18)30295-3

4. Xu XP, Yu XY, Wu X, et al. Propofol requirement for induction of unconsciousness is reduced in patients with Parkinson's disease: a case control study. Biomed Res Int. 2015;2015:953729. doi:10.1155/2015/ 953729 
5. Wang JF, Xu XP, Yu XY, et al. Remifentanil requirement for inhibiting responses to tracheal intubation and skin incision is reduced in patients with Parkinson's disease undergoing deep brain stimulator implantation. $J$ Neurosurg Anesthesiol. 2016;28(4):303-308. doi:10.1097/ANA.0000000000000229

6. Song D, Joshi GP, White PF. Titration of volatile anesthetics using bispectral index facilitates recovery after ambulatory anesthesia. Anesthesiology. 1997;87(4):842-848. doi:10.1097/00000542199710000-00018

7. White PF, Kehlet H, Neal JM, Schricker T, Carr DB, Carli F. The role of the anesthesiologist in fast-track surgery: from multimodal analgesia to perioperative medical care. Anesth Analg. 2007;104 (6):1380-1396. (). doi:10.1213/01.ane.0000263034.96885.e1

8. Song JG, Cao YF, Yang LQ, et al. Awakening concentration of desflurane is decreased in patients with obstructive jaundice. Anesthesiology. 2005;102(3):562-565. doi:10.1097/00000542200503000-00014

9. Wu Y, Jin S, Zhang L, et al. Minimum alveolar concentration-awake of sevoflurane is decreased in patients with end-stage renal disease. Anesth Analg. 2019;128(1):77-82. doi:10.1213/ANE.0000000000003676

10. Yang C, Feng Y, Wang S, et al. Effect of sex differences in remifentanil requirements for inhibiting the response to a $\mathrm{CO} 2$ pneumoperitoneum during propofol anesthesia: an up-and-down sequential allocation trial. BMC Anesthesiol. 2020;20(1):35. doi:10.1186/ s12871-020-0951-z

11. Katoh T, Ikeda $\mathrm{K}$, Bito $\mathrm{H}$. Does nitrous oxide antagonize sevoflurane-induced hypnosis? Br J Anaesth. 1997;79(4):465-468. doi:10.1093/bja/79.4.465

12. Bourgeois E, Sabourdin N, Louvet N, Donette FX, Guye ML, Constant I. Minimal alveolar concentration of sevoflurane inhibiting the reflex pupillary dilatation after noxious stimulation in children and young adults. Br J Anaesth. 2012;108(4):648-654. doi:10.1093/ bja/aer459

13. Niu B, Fang Y, Miao JM, et al. Minimal alveolar concentration of sevoflurane for induction of isoelectric electroencephalogram in middle-aged adults. Br J Anaesth. 2014;112(1):72-78. doi:10.1093/ bja/aet280

14. Cao Y, Zhang L, Peng X, et al. Increased minimum alveolar concentration-awake of Sevoflurane in women of breast surgery with sleep disorders. BMC Anesthesiol. 2020;20(1):17. doi:10.1186/ s12871-020-0931-3

15. Paul M, Fisher DM. Are estimates of MAC reliable? Anesthesiology. 2001;95(6):1362-1370. doi:10.1097/00000542-200112000-00014

16. Chen SQ, Ye HR, Chen YJ, Wang YW. MAC (EI) and MAC (awake) of sevoflurane in infants with obstructive jaundice. Paediatr Anaesth. 2014;24(3):282-289. doi:10.1111/pan.12322

17. Goo EK, Lee JS, Koh JC. The optimal exhaled concentration of sevoflurane for intubation without neuromuscular blockade using clinical bolus doses of remifentanil: A randomized controlled trial. Medicine. 2017;96(9):e6235. doi:10.1097/MD.0000000000006235

18. Munson ES, Hoffman JC, Eger EI 2nd. Use of cyclopropane to test generality of anesthetic requirement in the elderly. Anesth Analg. 1984;63(11):998-1000. doi:10.1213/00000539-198411000-00006

19. Gold MI, Abello D, Herrington C. Minimum alveolar concentration of desflurane in patients older than 65 yr. Anesthesiology. 1993;79 (4):710-714. doi:10.1097/00000542-199310000-00012
20. Sano T, Drummond JC, Patel PM, Grafe MR, Watson JC, Cole DJ. A comparison of the cerebral protective effects of isoflurane and mild hypothermia in a model of incomplete forebrain ischemia in the rat. Anesthesiology. 1992;76(2):221-228. doi:10.1097/00000542199202000-00011

21. Quasha AL, Eger EI 2nd, Tinker JH. Determination and applications of MAC. Anesthesiology. 1980;53(4):315-334. doi:10.1097/ 00000542-198010000-00008

22. Brian JE Jr, Bogan L, Kennedy RH, Seifen E. The impact of streptozotocin-induced diabetes on the minimum alveolar anesthetic concentration (MAC) of inhaled anesthetics in the rat. Anesth Analg. 1993;77(2):342-345. doi:10.1213/00000539-199377020-00022

23. Allaouchiche B, Duflo F, Debon R, Tournadre JP, Chassard D. Influence of sepsis on minimum alveolar concentration of desflurane in a porcine model. Br J Anaesth. 2001;87(2):280-283. doi:10.1093/ $\mathrm{bja} / 87.2 .280$

24. Emir UE, Tuite PJ, Oz G. Elevated pontine and putamenal GABA levels in mild-moderate Parkinson disease detected by 7 tesla proton MRS. PLoS One. 2012;7(1):e30918. doi:10.1371/journal.pone.0030918

25. Dharmadhikari S, Ma R, Yeh CL, et al. Striatal and thalamic GABA level concentrations play differential roles for the modulation of response selection processes by proprioceptive information. NeuroImage. 2015;120:36-42. doi:10.1016/j.neuroimage.2015.06.066

26. O'Gorman Tuura RL, Baumann CR, Baumann-Vogel H. Neurotransmitter activity is linked to outcome following subthalamic deep brain stimulation in Parkinson's disease. Parkinsonism Relat Disord. 2018;50:54-60. doi:10.1016/j.parkreldis.2018.02.014

27. Griffith HR, Okonkwo OC, O'Brien T, Hollander JA. Reduced brain glutamate in patients with Parkinson's disease. NMR Biomed. 2008;21(4):381-387. doi:10.1002/nbm.1203

28. Ishizeki J, Nishikawa K, Kubo K, Saito S, Goto F. Amnestic concentrations of sevoflurane inhibit synaptic plasticity of hippocampal CA1 neurons through gamma-aminobutyric acid-mediated mechanisms. Anesthesiology. 2008;108(3):447-456. doi:10.1097/ ALN.0b013e318164cfba

29. Sumikura H, Arendt-Nielsen L. MAC reduction after intrathecal coadministration of GABA (A) agonist and glutamate antagonist in rats. J Anesth. 2003;17(2):92-97.

30. Antkowiak B. Different actions of general anesthetics on the firing patterns of neocortical neurons mediated by the GABA (A) receptor. Anesthesiology. 1999;91(2):500-511. doi:10.1097/00000542199908000-00025

31. Johnston RR, Way WL, Miller RD. The effect of CNS catecholamine-depleting drugs on dextroamphetamine-induced elevation of halothane MAC. Anesthesiology. 1974;41(1):57-61. doi:10.1097/00000542-197407000-00013

32. Nakao H, Ono J, Nogaya J, Yokono S, Yube K. The relationship of brain catecholamine levels to enflurane requirements among three strains of mice with different anesthetic sensitivities. $J$ Anesth. 2001;15(2):88-92. doi:10.1007/s005400170033

33. Segal IS, Walton JK, Irwin I, et al. Modulating role of dopamine on anesthetic requirements. Eur $J$ Pharmacol. 1990;186(1):9-15. doi:10.1016/0014-2999(90)94055-3

34. Abdelmawgoud A, Rashwan S. Effect of preoperative oral amantadine on intraoperative anesthetic and analgesic requirements in female patients during abdominoplasty. Egyptian $J$ Anaesthesia. 2019;29(1):7-11. doi:10.1016/j.egja.2012.09.001 


\section{Publish your work in this journal}

Clinical Interventions in Aging is an international, peer-reviewed journal focusing on evidence-based reports on the value or lack thereof of treatments intended to prevent or delay the onset of maladaptive correlates of aging in human beings. This journal is indexed on PubMed Central, MedLine, CAS, Scopus and the Elsevier
Bibliographic databases. The manuscript management system is completely online and includes a very quick and fair peer-review system, which is all easy to use. Visit http://www.dovepress.com/ testimonials.php to read real quotes from published authors.

Submit your manuscript here: https://www.dovepress.com/clinical-interventions-in-aging-journal 